\title{
INTERNAL CONSISTENCY OF A BRAZILIAN VERSION OF THE UNIFIED HUNTINGTON'S DISEASE RATING SCALE
}

\author{
Vitor Tumas¹, Sarah Teixeira Camargos², Paulo Samandar Jalali², \\ Adriano de Paula Galesso², Wilson Marques J ${ }^{3}$
}

\begin{abstract}
We evaluated the reliability of a translated Brazilian version of the Unified Huntington'sDisease Rating Scale (UHDRS) to establish the reproducibility of the scale in a population that differs substantially from that on which the scale was originally validated. After a training period with the video and guidelines requested from the Huntington Study Group, we applied the UHDRS, except for the cognitive tests, to a group of 21 Brazilian patients with a molecular diagnosis of Huntington's disease (HD). We found a high degree of internal consistency of the motor section of the UHDRS (Cronbach's alpha $=0.841$ ). There was a negative correlation between the total motor score and the functional assessment, the independence scale and the functional capacity. There was a positive correlation between these 3 scales of functional evaluation and a negative correlation between the age of onset of the disease and the number of CAG repeats. The behavioral scale and disease duration were not correlated with any factor. The clinical characteristics of this sample of patients as described by the UHDRS were roughly similar to those reported in the original validation studies and the correlations described were similar to those reported previously. We conclude that the Brazilian version of the UHDRS is reliable and valid to study patients with HD in the Brazilian setting, that this sample of Brazilian patients had clinical characteristics similar to those observed in other world regions, as expected, and that the clinical training method used for the application of the UHDRS was effective to insure a high degree of clinical reproducibility.
\end{abstract}

KEY WORDS: Huntington's disease, Unified Huntington's disease rating scale, Brazilian version, reliability.

\begin{abstract}
Consistência interna da versão brasileira da escala unificada para avaliação da doença da Huntington
RESUMO - Nosso estudo avaliou a confiabilidade da versão brasileira da escala unificada para avaliação da doença de Huntington (UHDRS) com o objetivo de estabelecer a reprodutibilidade dessa escala em uma população que difere significativamente daquela em que foi originalmente validada. Após um período de treinamento com um vídeo divulgado para esse objetivo e um manual solicitado aosidealizadores da escala, nós aplicamos a UHDRS, com exceção dos testes cognitivos, em um grupo de 21 pacientes brasileiros com diagnóstico molecular de doença de Huntington (DH). Nessa amostra nós encontramos uma elevada consistência interna na seção que avalia os sintomas motores (alfa de Cronbach=0,841). Observamos uma correlação negativa entre o escore total da escala motora e as escalas de avaliação funcional, de independência e de capacidade funcional total; uma correlação positiva entre essas 3 últimas escalas; e uma correlação negativa entre a idade de início dossintomas e o número de repetições CAG. As características clínicas dessa amostra de pacientes descritas segundo a UHDRS é de maneira comparativa similar às descritas nos estudos originais de validação da escala, assim como as correlações descritas são semelhantes às descritas previamente. Concluimos que a versão brasileira da UHDRS é confiável e válida para estudar pacientes com $\mathrm{DH}$ aqui no Brasil, que essa amostra, como era esperado, tem características semelhantes às descritas em outras regiões do mundo, e que o método utilizado para treinamento da aplicação da escala é válido.
\end{abstract}

PALAVRAS-CHAVE: doença de Huntington, escala unificada, versão brasileira, confiabilidade.

Huntington's disease (HD) isa hereditary neurodegenerative disease with worldwide distribution resulting from a CAG repeat expansion in the IT15 gene located on $4 \mathrm{p} 16.3^{1}$. The progressively com- plexclinical picture includesmotor manifestations, psychiatric symptoms and cognitive abnormalities $^{2}$. Abnormal movements are one of the most frequent motor problemsthat also include gait and

Department of Neurology, Psychiatry and Medical Psychology, Ribeirão Preto School of Medicine, University of São Paulo, Ribeirão Preto SP, Brazil: ${ }^{1}$ Head of the Movement Disorders Section, ${ }^{2}$ Resident in Neurology, ${ }^{3}$ Associate Professor.

Received 25 March 2004. Accepted 17 July 2004.

Dr. Vitor Tumas - Department of Neurology, Psychiatry and Medical Psychology - Ribeirão Preto School of Medicine, University of São Paulo - 14049-900 Ribeirão Preto SP - Brasil. E-mail: tumasv@rnp.fmrp.usp.br 
postural disturbances, oculomotor abnormalities, changes in muscular tonus and bradykinesia. It seems that the disease originated and was more frequent in Caucasians, and was probably disseminated throughout the world by European migrations in the 17th and 18th centuries ${ }^{2}$. Accordingly, the same CAG repeat expansion is identified in patients all over the world ${ }^{3}$. New mutations are rare, but they can occur due to the instability in the paternal transmission of an intermediate allele with 27-35 CAG repeats ${ }^{4}$. It is well accepted that the phenotypical aspects of the disease are very similar in different populations. The molecular aspects of Brazilian HD patients have been recently reported and were found to be similar to the findings obtained for other ethnic groups $s^{5,6}$. Consequently we expect the phenotypical presentation of these patients to be similar to the classical descriptions.

The Unified Huntington's Disease Rating Scale (UHDRS $^{7}$ is a clinical rating scale developed to standardize the clinical examination of HD patients, scoring in detail the four main domains of impairment: motor performance, behavior abnormalities, cognitive performance and functional capacity. Its reliability and consistency have been dearly demon- strated in North-American patients ${ }^{7}$, but so far no study has been reported to validate the scale in other ethnic groups. After translating the UHDRSinto Portuguese we applied it to a group of Brazilian patientsfollowed at our university hospital to determine the reliability of the translated scale and to establish its reproducibility in a population that differs substantially from that where it was originally studied in terms of linguistic and cultural aspects. We also intended to determine if the phenotypical characteristics of this sample of Brazilian HD patients are similar to the classical descriptions throughout the world, and if this small sample is a representative cross-sectional view of the disease.

\section{METHOD}

We have included in thisstudy all patientswith a molecular diagnosis of Huntington's disease followed at the Movement Disorder outpatient dinic of our university hos pital, State of São Paulo, Brazil. All patients and parents gave informed consent for blood and clinical analysis, and the protocol was approved by the local ethics committee.

For molecular diagnosis, DNA was extracted from peripheral leucocytes according to standard protocols. The pertinent region of chromosome $4 p$ was expanded by $\mathrm{PCR}$ with primers $\mathrm{HD} 3 \mathrm{~F}$ and $\mathrm{HDE}$, as previously described ${ }^{8}$.

Table 1. Characteristics of 21 Brazilian patients with Huntington's disease according to the UHDRS.

\begin{tabular}{lccc}
\hline & Mean & SD & Range \\
\hline Age at examination & 46.57 & 13.00 & $25-70$ \\
Age at HD onset & 38.71 & 13.22 & $16-61$ \\
Male/female & $8 / 13$ & & \\
In use of neuroleptics & $16 / 21$ & & \\
Duration of HD (years) & 7.85 & 4.50 & $2-20$ \\
Abnormal CAG repeats & 46.95 & 5.21 & $41-58$ \\
Total motor score (0-124) & 46.55 & 23.90 & $17-89$ \\
Total behavior score (0-224) & 22.66 & 25.58 & $0-75$ \\
Behavioral milestones & & & \\
\multicolumn{1}{c}{ Confused (\%) } & $38 \%$ & & \\
$\quad$ Demented (\%) & $33 \%$ & & \\
\multicolumn{1}{c}{ Depressed (\%) } & $42 \%$ & & \\
Requiring an antidepressive (\%) & $33 \%$ & & $0-12$ \\
Functional checklist score (0-25) & 10.71 & 7.47 & \\
Independence scale (0-100\%) & $65.23 \%$ & $25.61 \%$ & \\
Total functional capacity (0-13) & 5.61 & 4.00 & \\
\hline
\end{tabular}


PCR productswere electrophoresed in an automated 377 Sequencer and analyzed with the Genescan software. Individuals with more than 36 CAG repeats were considered to have a positive molecular diagnosis of Huntington's Disease ${ }^{4}$.

The UHDRSguidelines and the videotape of the motor examination were obtained from the Huntington Study Group 7 . After being adapted and translated to Brazilian patients by one of the authors(VT) they were used in training sessions with all four clinical investigators (VT, STC, APG, PS ) until a consensus about the examination technique and the grades of each motor abnormality was reached. At the end of the training period a reasonably uniform agreement about score rate was obtained from the 4 investigators. The interrater reliability of the translated version will be accessed in another study. After this period of training all patients were evaluated by the same investigator (STC), who used the adapted version of the UHDRS to score the motor, behavioral and functional assessments, the independence scale and the functional capacity score. The cognitive assessment were not applied in this study due to the wide variations in the cultural and educational background of the patients, and to the absence of validation studies for the cognitive tests in our normal population. The Luria test was also excluded from the analysis because it was not rated in many patients for different reasons.

In the UHDRS, the motor performance is described by the total motor score which is calculated by adding the scores for each of the 15 items of motor function (grading $0=$ normal to $4=$ severely impaired). Many of these items are scored in more than one condition or body region to make up a total of 33 scores to be added to achieve the worst performance of 132. The exclusion of the Luria test resulted in a maximum possible motor score of 128 . The remaining 14 items of the motor exam- ination were grouped into 9 dimensions for statistical analysis as follows: 1) abnormalities of ocular motor control (ocular pursuit, saccade initiation, saccade velocity) 2) dysarthria, 3) motor impersistence (tongue protrusion), 4) bradykinesia (finger taps, pronate-supinate hands, body bradykinesia), 5) rigidity, 6) dystonia, 7) chorea, 8) abnormalities of gait (gait, tandem gait), and 9) postural instability. The dimensions were established by their clinical contentsand not by previousscale principal component analysis.

The behavioral assessment of the UHDRS is calculated by adding different items, and each one is defined by the product of the severity ( $0=$ absent to $4=$ severe) and the frequency ( $0=$ almost never, 4 almost always) of the psychiatric symptom. In another session, three items were used to assess if the patient has reached certain behavioral milestones (depression, dementia, confusion) according to the examiner. The cognitive performance that was not investigated in our study was described in the scale by verbal fluency, symbol digit and Stroop tests. The score on the functional assessment scale was the sum of 25 daily tasksthat the patient could still perform. The independence scale ranged from 100 (no special care needed) to 10 (tube feeding, total bed care). The functional capacity was the sum of 5 items with a total score of 0 (unable) to 13 (normal). Thus, the functional scales score higher for better patient condition, this being the inverse of the other subscales.

The number of abnormal CAG repeats, age at disease onset and duration of disease were recorded. We analyzed the internal consistency of the motor scale data using Cronbach's alpha and calculated an item-total and item-item correlation.

We also studied the correlations between total motor score, behavioral score, functional assessment, independence scale, functional capacity score, number of abnor-

Table 2. Analysis of the reliability of the motor scale of the UHDRS: correlation matrix of item-total and item-item correlation.

\begin{tabular}{|c|c|c|c|c|c|c|c|c|c|c|}
\hline & TOTAL & OMC & DYSART & TONGUE & BRADY & RIGID & DYST & $\mathrm{CHOR}$ & GAIT & RETR \\
\hline TOTAL & 1.00 & & & & & & & & & \\
\hline OMC & 0.776 & 1.00 & & & & & & & & \\
\hline DYSART & 0.890 & 0.704 & 1.00 & & & & & & & \\
\hline TONGUE & 0.870 & 0.792 & 0.853 & 1.00 & & & & & & \\
\hline BRADY & 0.910 & 0.818 & 0.877 & 0.842 & 1.00 & & & & & \\
\hline RIGID & 0.527 & 0.420 & 0.505 & 0.641 & 0.566 & 1.00 & & & & \\
\hline DYST & 0.645 & 0.721 & 0.814 & 0.738 & 0.769 & 0.545 & 1.00 & & & \\
\hline CHOR & 0.435 & 0.379 & 0.501 & 0.427 & 0.499 & 0.139 & 0.115 & 1.00 & & \\
\hline GAIT & 0.906 & 0.710 & 0.824 & 0.845 & 0.885 & 0.577 & 0.642 & 0.592 & 1.00 & \\
\hline RETR & 0.823 & 0.605 & 0.685 & 0.722 & 0.775 & 0.544 & 0.475 & 0.633 & 0.890 & 1.00 \\
\hline
\end{tabular}

TOTAL, total motor score; OMC, abnormalities of ocular motor control; DYSART, dysarthria; TONGUE, motor impersistence; BRADY, bradykinesia; RIGID, rigidity; DYST, dystonia; CHOR, chorea; GAIT, abnormalities of gait; RETR, postural instability 


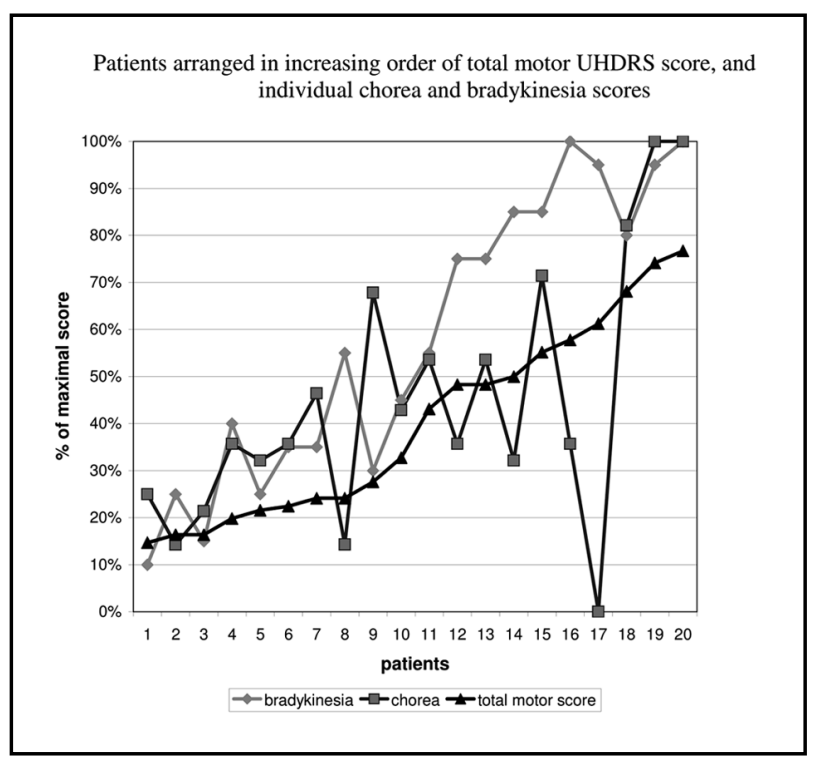

Fig 1. Graphic showing the $21 \mathrm{HD}$ patients arranged in crescent order of their total motor score, the unit is the percentage of the maximal possible motor score. At the same way the scores of 2 sub-items, chorea and bradykinesia of the motor examinations of each patient were also presented. Bradykinesia and chorea were respectively the most and the less correlated items with the total motor score.

mal CAG repeats, age at disease onset, and disease duration using the Pearson's correlation matrix.

The working hypothesis was that the dinical characteristicswould be similar to those of the patients described in the original UHDRS study and that the translated version would be reliable, validating the Brazilian version of the UHDRS.

\section{RESULTS}

We examined 21 patients from 20 families with a molecular diagnosis of HD who were followed at our Hospital. At the time of evaluation, most of them were taking neuroleptics. Table 1 shows the demographic data and the clinical characteristics of this group of patients according to the UHDRS, except for the cognitive section and the Luria test. An item-total correlation was calculated between the scoresfor each of the 9 dimensions of the motor examination and the total motor score, and we also calculated an item-item correlation (Table 2).

We found that chorea (0.435), rigidity (0.527) and dystonia (0.645) were the clinical dimensions less correlated with the total motor score and also with the other items. The dimensions which were less correlated were rigidity and chorea (0.139) and chorea and dystonia (0.115). The most correlated clinical dimensions were postural instability and gait (0.890), bradykinesia and gait (0.885) and bradykinesia and dysarthria (0.877)
The Cronbach's alpha value of the motor section of the UHDRSwas 0.841 , which indicates a high degree of internal consistency. The variation in alpha value, if each item was deleted from the motor scale, showed that the exclusion of the chorea dimension from the motor scale wasthe only one that resulted in an increase of Cronbach's alpha (0.893), while the exclusion of the bradykinesia dimension resulted in the greatest reduction in the scale alpha value (0.786).

To show that the total motor score represents a linear curve of the theoretical construct of the motor examination, as was expected by its high internal consistency, we graphically plotted the total motor scores of the 21 patients in increasing order. The scores were transformed to the percentage of the total motor score (Fig 1). Furthermore, in the same graphic and using the same methodology we presented the 2 sub-items of motor examination of each patient, chorea and bradykinesia (Fig 1). As can be seen, the total motor score presented an almost linear ascending curve and the 2 dimensions of the motor examination followed a more irregular course. The chorea curve followed a more irregular ascending course than the bradykinesia curve asit wasthe least correlated item to the total motor score.

The Pearson correlation matrix showed a negative correlation between total motor score and functional assessment $(-0.709, p<0.001)$, independence scale $(-0.745, p<0.001)$ and functional capacity ($0.686, p \varangle 0.001$ ), a positive correlation between the 3 scales of functional evaluation $(p<0.001$ ), and a negative correlation between the age at onset of the disease and the number of CAG repeats $(-0.695$, $p<0.0001$ ). The behavioral subscale and disease duration were not correlated with any factor.

\section{DISCUSSION}

The UHDRS is a reliable clinical rating scale developed to assess motor performance, cognitive functioning, behavioral abnormalities and functional capacity of HD patients ${ }^{7,9}$. It may be used as a tool for evaluating the progression of the disease and so for determining the natural history of the disease and the effects of therapeutic interventions ${ }^{10}$.

We have adapted the UHDRS to Brazilian patientstaking care to preserve itsoriginal purpose and obtained many indications of its reliability in this study. It is supposed that cultural and linguistic characteristics of different populations may alter the results obtained with a clinical scale developed 
for a specific ethnic group, so that the validation and reliability of a clinical rating scale may not be the same across different populations ${ }^{11}$. Accordingly, it is always important to validate the scale in populations that differ substantially from the original one in terms of linguistic and cultural aspects ${ }^{11}$. In the present study we found in this study a high internal consistency in the motor scale of the UHDRS when the adapted version was applied to a Brazilian sample of $21 \mathrm{HD}$ patients (Cronbach's alpha $=0.893$ ). This result is very similar to that described in the original validation study ${ }^{11}$. Cronbach's al pha may be interpreted as the average correlation between two halves of a test when the test is split into all possible combinations of two half tests ${ }^{11}$. A high alpha indicates that there is at least one homogeneous dimension underlying the summed score and that at least some of the items correlate substantially with one another ${ }^{11}$. In clinical settings, an alpha between 0.80 and 0.90 ought to be the goal ${ }^{11}$.

There are several other methods available to evaluate the internal consistency or reliability of a linear composite scale as the UHDRS. In item-total correlation, each item is correlated with the sum of the remaining items. In our study almost all items of motor examination showed high item-total correlations except for chorea, rigidity and dystonia in increasing order of correlation with the total motor score. Also, chorea showed very low correlations with most of the other examination items and if excluded from the scale resulted in an increase in itsinternal consistency. This seems paradoxical in the case of HD where chorea is one of the main motor symptoms and should be an obligate participant in the theoretical construct of a clinical rating scale for the disease. The possible explanation for thisfinding wasthat almost all of our patients were examined while taking neuroleptics to control abnormal movements. This could be an indicator that in our medical setting there is a tendency to prescribe freely these medications even to patients with mild chorea. The low correlation between chorea and rigidity may be related to the fact that the UHDRS does not measure tonus properly, it would be also necessary to include measures of hypotonia in the motor scale. Figure 1 which represents each patient ordered according to total motor score is a useful illustration that the sample spans the spectrum of the illness and that this straight ascending line would be expected if the scale measured a linear construct. Cons- tructs are abstract concepts about the disease that are materialized in a number of observable indicators of the abstraction ${ }^{11}$. An ordinal composite scale such as the UHDRS may follow the model of a linear composite scale, where, when the items are summed, the trace line plotting total scores against the construct being measured will approximate a straight line. This is expected in a linear model of a construct that measures the motor symptoms of a disease. The individual items tend to have a slightly different curve when plotted against the construct. We can see in Figure 1 that the patient's total motor score ranged from $10 \%$ to $80 \%$ of the total possible score, and therefore we may speculate that our small sample correspondsto a valid cross-sectional clinical representation of HD patients.

We detected a very low correlation between chorea and dystonia in our patients, supporting other observationsthat indicate that these motor abnormalitiesmay evolve separately ${ }^{12}$. The most correlated clinical dimensions in our study were postural instability and gait (0.890), bradykinesia and gait (0.885) and bradykinesia and dysarthria (0.877). This may indicate that these clinical aspects measure a homogeneous clinical factor with related patophysiological mechanisms.

The high correlation between the total motor score and the functional scalesthat were also highly interrelated, and the absence of a correlation between the behavioral assessment and the other subscales are findings similar to those reported by others ${ }^{7}$. This indicates that the behavioral symptomsfollow a separate and independent patophysiological mechanism or that the neuroleptical therapy would be interfering with the clinical presentation of these symptoms, since most of the patients were in use of these drugs. Also, the negative correlation between the age at onset of the disease and the number of CAG repeats observed in our study is similar to that detected in many other studies. These findings corroborate the reproducibility of the scale and of the HD clinical manifestations in our medical setting.

Another important point in this study was the strong indication of the validity of the training method for the application of the scale. If we had introduced any systematic error in our medical evaluation because of some consistently biasing factor such as an erroneousinterpretation of the clinical examination due to a misunderstanding of the scale items or of the examination technique, 
we should have expected a low internal consistency. Internal consistency is a form of reliability, i. e. the proportion of variation in scores due to true variation and not to error, and its measure is sensitive to unreliability attributable to content sampling as error variance ${ }^{11}$.

Finally, if we compare the clinical and UHDRS characteristics of our sample of Brazilian HD patients to those of the other validation studies with a large number of North-American and Dutch patients ${ }^{7,9}$, we can see that our population is closely similar. The most obvious difference compared to the first study ${ }^{7}$ is that confusion is more frequent $(38 \%)$ in our population (17\%). Because we are comparing only the global score of the motor examination we cannot exclude that some differences in the phenotypical presentation of the disease may exist. But these possible differences could also be an effect of the small size of our sample. Excluding the Luria test of this analysis seems to have had a small effect on the total motor score. It is a component of the motor scale and it scores 4 at worst performance, and this could not change significantly the total motor score of the sample.

We may conclude that the Brazilian version of the UHDRS is reliable and valid for the study of patients with HD in the Brazilian setting and that the clinical training method used for the application of the UHDRS was effective to insure a high degree of clinical reproducibility. We also conclude that, as expected, the phenotypical presentation of HD in our country is similar to that in other world regions.

\section{REFERENCES}

1. Huntington's Disease Collaborative Research Group. A novel gene containing a trinucleotide repeat that is expanded and unstable on Huntington's disease chromosomes. Cell 1993;72:971-983.

2. Bruyn GW, Went LN. Huntington's chorea. In: Vinken PJ, Bruyn GW, Klawans HL (eds). Handbook of clinical neurology vol 49, Extrapyramidal Disorders. Amsterdam: Elsevier Science Publishers, 1986:267-313.

3. Kremer B, Goldberg P, Andrew SE, et al. A worldwide study of the Huntington's disease mutation: the sensitivity and specificity of measuring CAG repeats. N Eng J Med 1994;330:1401-1406.

4. American College of Medical Genetics, American Society of Human Genetics, Huntington Disease Genetic Testing Working Group. Laboratory guidelines for Huntington disease testing. Am J Hum Genet 1998;62:1243-1247.

5. Silva TCL, Serra HG, Bertuzzo CS, Lopes-Cendes IL. Molecular diagnosis of Huntington disease in Brazilian patients. Arq Neuropsiquiatr 2000;58:11-17.

6. Raskin S, Allan N, Teive HG, et al. Huntington disease: DNA analysis in Brazilian population. Arq Neuropsiquiatr 2000;58:977-985.

7. Huntigton Study Group. Unified Huntington's disease rating scale: reliability and consistency. Mov Disord 1996;11:136-142.

8. Davis MB, Bateman DM, Quinn NP, Marsden CD, Harding AE. Mutation analysis in patients with possible but apparently sporadic Huntington's disease. Lancet 1994;344:714-717.

9. Siesling S, Zwinderman KAH, Van Vugt JPP, Kieburtz K, Roos RAC. A shortened version of the motor section of the unified Huntington's disease rating scale. Mov Disord 1997;12:229-234.

10. Siesling S, Van Vugt JPP, Zwinderman KAH, Kieburtz K, Roos RAC. Unified Huntington's disease rating scale: a follow up. Mov Disord 1998;13:915-919.

11. Larocca NG. Statistical and methodological considerations in scale construction. In: Munsat TL (ed). Quantification of neurologic deficit. Boston: Butterworths, 1989:49-67.

12. Louis ED, Anderson KE, Moskowitz C, Thorne DZ, Marder K. Dystoniapredominant adult-onset Huntington disease. Arch Neurol 2000;57:1326-1330. 